\title{
Evaporation-Induced Biomolecule Detection on Versatile Superhydrophilic Patterned Surfaces: Glucose and DNA Assay
}

\author{
Pinar Beyazkilic, Abtin Saateh, Mehmet Bayindir, and Caglar Elbuken*(i)
}

UNAM - National Nanotechnology Research Center and Institute of Materials Science and Nanotechnology, Bilkent University, 06800 Ankara, Turkey

\section{Supporting Information}

ABSTRACT: We introduce a droplet-based biomolecular detection platform using robust, versatile, and low-cost superhydrophilic patterned superhydrophobic surfaces. Benefitting from confinement and evaporation-induced shrinkage of droplets on wetted patterns, we show enrichment-based biomolecular detection using very low sample volumes. First, we developed a glucose assay using fluorescent polydopamine (PDA) based on enhancement of PDA emission by hydrogen peroxide $\left(\mathrm{H}_{2} \mathrm{O}_{2}\right)$ produced in enzyme-mediated glucose oxidation reaction. Incubation in evaporating droplets resulted in brighter fluorescence compared to that in bulk solutions. Droplet assay was highly sensitive toward increasing glucose concentration while that in milliliter-volume solutions resulted in no fluorescence enhancement at similar time scales. This is due to droplet evaporation that increased the reaction rate by causing enrichment of PDA and glucose/glucose oxidase as well as increased concentration of $\mathrm{H}_{2} \mathrm{O}_{2}$ generated in shrinking droplet. Second, we chemically functionalized wetted patterns with single-stranded DNA and developed fluorescence-based DNA detection to demonstrate the adaptability of the patterned surfaces for a different class of assay. We achieved detection of glucose and DNA with concentration down to $130 \mu \mathrm{M}$ and 200 $\mathrm{fM}$, respectively. Patterned superhydrophobic surfaces with their simple production, sensitive response, and versatility present potential for bioanalysis from low sample volumes.

\section{INTRODUCTION}

Superhydrophobic surfaces patterned with well-defined wetted patterns have been important for high-throughput biological and chemical screening inside droplets placed on top since they provide increased reaction rates and sensing signals. ${ }^{1}$ Microscale droplets are confined on superhydrophilic patterns due to wetting contrast between hydrophilic patterns and hydrophobic areas. ${ }^{2}$ Upon evaporation of droplets, substances dispersed in droplets enrich, which increases molecular interaction frequencies. Such an enrichment effect increases reaction rates, providing enhanced detection signals, whereas signals remain too low to be detected in low-concentration samples. ${ }^{3}$ A few reports have demonstrated detection of DNA, RNA, protein, and bacteria inside stationary droplets by exploiting high sensitivity advantage of evaporation-induced enrichment effect. ${ }^{3-8}$ Here, to obtain a practical platform for droplet-based assays, we present the use of superhydrophobic organically modified silica-coated surfaces with circular superhydrophilic patterns generated by converting surface-bound methyl groups into hydroxyl groups via UV/ozone treatment. Two-dimensional chemical patterning without physical decomposition resulted in isolation of aqueous droplets in small wetted spots (Figure 1). These surfaces provide a very strong contrast between wetting and nonwetting regions. Additionally, they can be generated with high precision and they are suitable for large-area processing and stable at a wide range of temperature. ${ }^{9,10}$ Here, we show the versatility of these surfaces using two types of assays, which are based on different chemistries, a novel glucose sensing assay and a DNA hybridization assay.

Hernandez-Perez et al. have recently developed a micropillar-based platform and have shown that $2.5 \mathrm{mM}$ glucose can be detected in evaporating droplets placed on pillars. They used horseradish peroxidase (HRP)/glucose oxidase (GOx)/ chromogen as the sensing probe. ${ }^{11}$ However, HRP has some drawbacks, such as high cost and instability. Therefore, various peroxidase-mimicking nanomaterials have been introduced, including quantum dots, metal nanoclusters, and dyes. Conjugated polymers have also been used as peroxidasemimicking nanomaterials based on direct or indirect interactions between $\mathrm{H}_{2} \mathrm{O}_{2}$ and polymer backbones. ${ }^{12,13}$ Although such materials exhibit high sensitivity for glucose detection, they usually involve complicated synthesis steps ${ }^{14,15}$ and toxicity. Contrary to these materials, polydopamine (PDA) can be easily produced through oxidation and self-polymerization of dopamine in alkaline environments. Particularly, PDA fluorescence has recently found applications in bioimaging and dopamine sensing. ${ }^{16-18}$ Here, we used

Received: March 3, 2018

Accepted: July 16, 2018

Published: October 18, 2018 


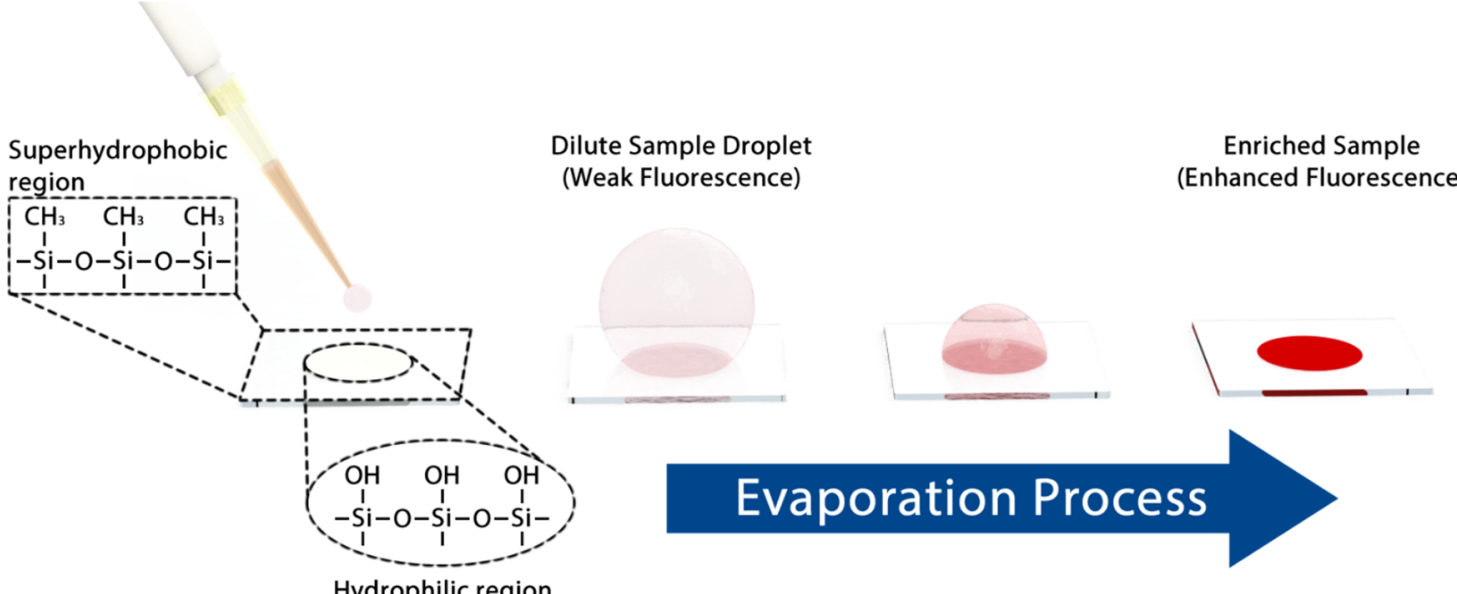

Figure 1. Schematic representation of droplet pinning on superhydrophilic circular pattern of a superhydrophobic ormosil surface and evaporationinduced enrichment of low-concentration, low-volume fluorescent sample and resulting fluorescence enhancement.
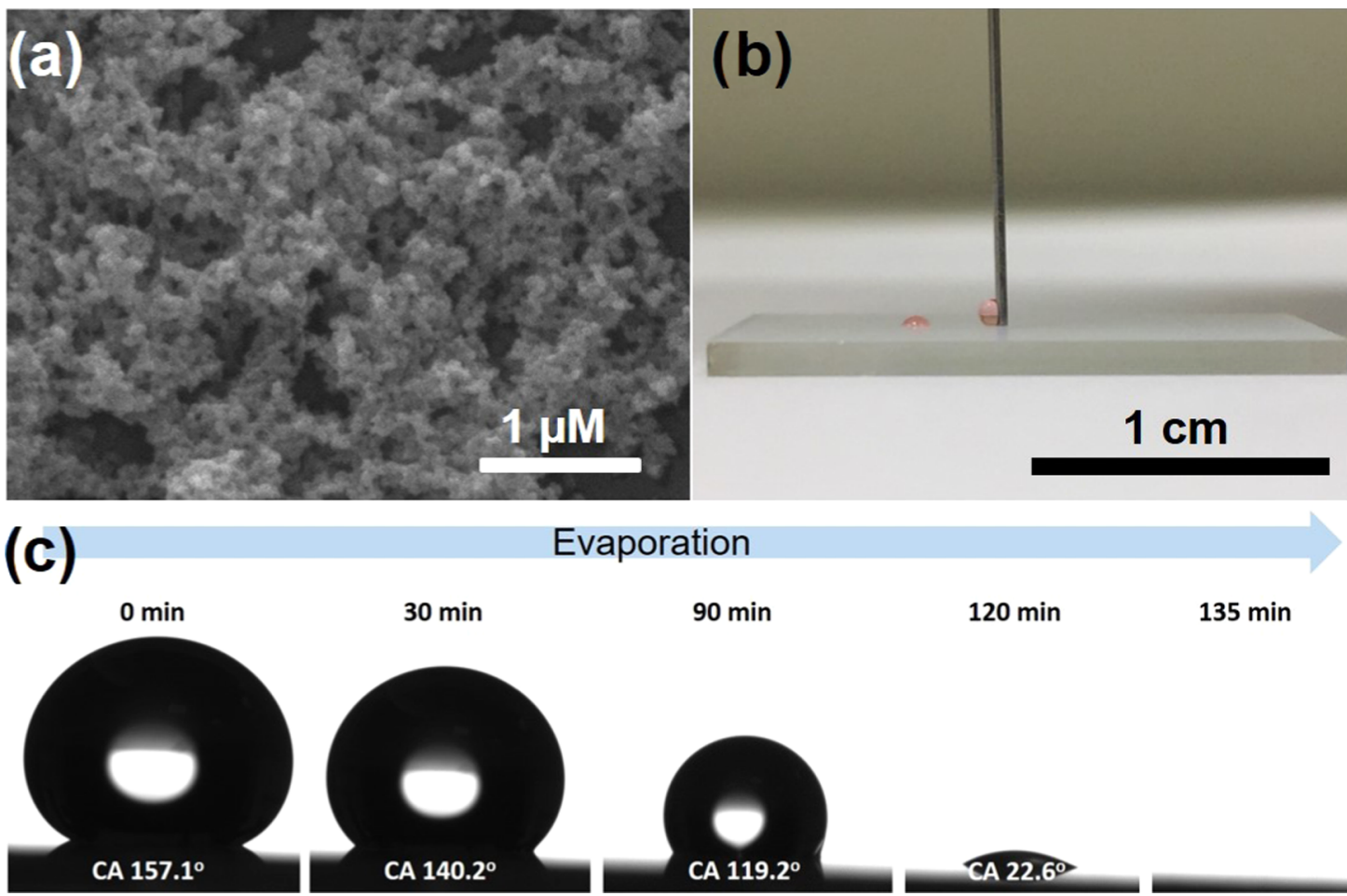

Evaporation

$90 \mathrm{~min}$

$120 \mathrm{~min}$

$135 \mathrm{~min}$

Figure 2. (a) Scanning electron microscopy image of patterned superhydrophobic silica surface. (b) Water droplet dyed with Ponceau 4R, a food additive, on wetted spot and nonwetted region of patterned surface. Droplet was confined on the wetted area, whereas it was repelled from the nonwetted part and maintained its spherical shape due to low surface energy. (c) Time-dependent contact angle profile of a water droplet on superwetted pattern at room temperature.

fluorescent PDA for droplet-based glucose sensing probe, which is another major contribution of this work. A sensitive glucose assay was prepared by combining the advantages of PDA, such as biocompatibility and ease of production with analyte/product-enriching capability of patterned surfaces.

PDA fluorescence is known to enhance due to decomposition of its stacked chain structure by $\mathrm{H}_{2} \mathrm{O}_{2}$, which is produced in glucose oxidation reaction. To the best of our knowledge, we have exploited PDA as glucose probe for the first time. We first produced PDA from dopamine and incubated with glucose/GOx inside microdroplets placed on wetted patterns. After droplet evaporation, aggregated PDA on the patterns radiated bright fluorescence, which was observed to increase with increasing glucose concentration. However, when incubated in milliliter-volume bulk solution, no significant fluorescence enhancement was obtained within the same time $(\sim 50 \mathrm{~min})$. Fast fluorescence enhancement and stronger signals on patterns revealed increase of reaction rate and sensitivity in evaporating droplets.

As a second assay geared toward molecular diagnostics, we functionalized wetted patterns with single-stranded DNA (ssDNA) and performed fluorescence-based DNA sensing in evaporating microdroplets. Hybridization of target and probe DNA strands inside droplets resulted in higher fluorescence intensities compared to milliliter-volume solution, where no enrichment occurred. Fluorescence of hybridized DNA with 
(a)

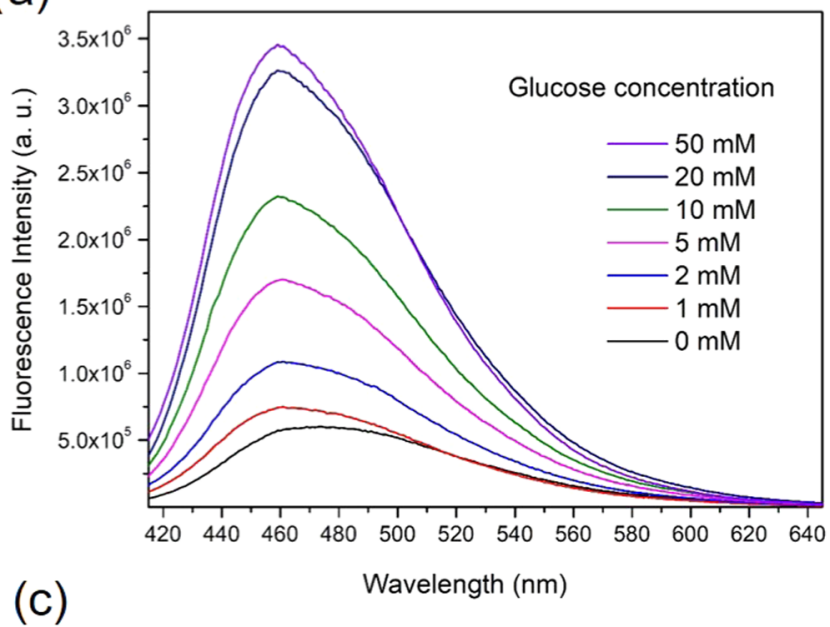

(b)

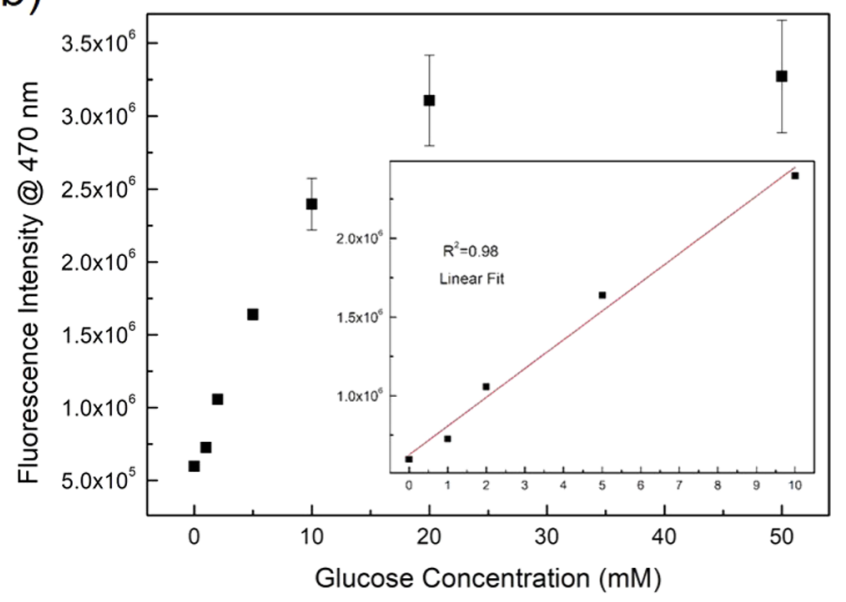

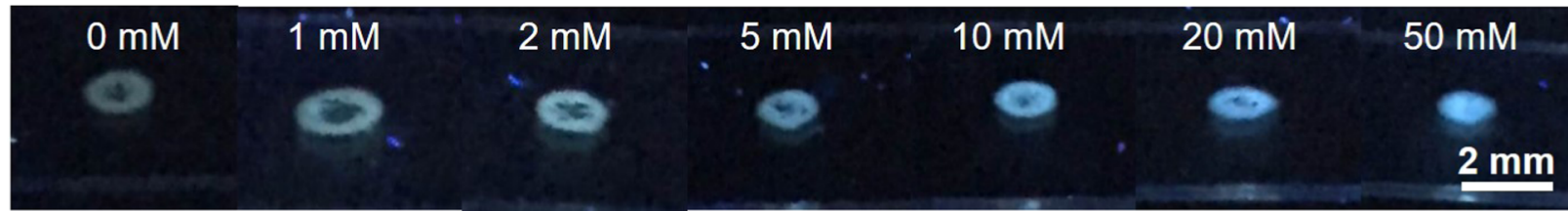

Figure 3. (a) Fluorescence spectra of PDA incubated with glucose with concentration ranging from 1 to $50 \mathrm{mM}$ in evaporating microdroplets on wetted patterns. (b) Fluorescence intensities at $470 \mathrm{~nm}$ plotted with data in (a). The inset shows linear calibration curve in the range of 1-10 mM. The error bars show 1 standard deviation of three measurements. (c) Photographs of fluorescent PDA spots excited by UV light (366 nm) after incubation with glucose in evaporating droplets.

concentration as low as $200 \mathrm{fM}$ was detected thanks to the enrichment effect of patterned surfaces.

\section{RESULTS AND DISCUSSION}

Superhydrophilic-superhydrophobic patterned surfaces were produced using a one-pot sol-gel method, followed by UV/ ozone treatment on the positions defined (see Materials and Methods section for details). Superhydrophobic coatings were composed of methyl $\left(\mathrm{CH}_{3}\right)$-modified nanoparticles, which were organized in a porous and rough network (Figure 2a). The contact angle of superhydrophobic surfaces was $169^{\circ}$ (Figure S1). Superwetted spots with diameter of 0.9 or $1.4 \mathrm{~mm}$ were generated on as-prepared superhydrophobic surfaces. To obtain these spots, surfaces were aligned under a shadow mask with holes formed by laser cutting system and treated with $\mathrm{UV} /$ ozone, which decomposed hydrophobic $\mathrm{CH}_{3}$ groups, leaving silanol $(\mathrm{Si}-\mathrm{OH})$ groups. ${ }^{4}$ Extreme wettability difference between untreated and UV/ozone-treated regions led to confinement of microdroplets and solute substances on small wetted patterns, while droplets were repelled from untreated area due to superhydrophobicity (Figure $2 b$ ). The degree of wettability was tuned depending on UV/ozone treatment time. The contact angle gradually decreased with increasing treatment time and surface became superhydrophilic upon 40 min treatment (Figure S2). To obtain evaporation-induced droplet confinement, time-dependent contact angle on a pattern was measured. A $20 \mu \mathrm{L}$ droplet drop-cast onto pattern was initially quasi-spherical due to the superhydrophobicity of the surrounding pattern (Figure 2c). Upon evaporation, the contact angle gradually decreased due to reduced volume and the droplet was finally evaporated.

We incubated PDA with glucose solution with concentration ranging from 1 to $50 \mathrm{mM}$ in $20 \mu \mathrm{L}$ of evaporating droplets (see Materials and Methods section for details). Fluorescence intensities were recorded after droplets were evaporated, which took approximately $50 \mathrm{~min}$ at $37{ }^{\circ} \mathrm{C}$. PDA, which is formed through oxidation and polymerization of dopamine, yields weak green broad-band fluorescence in the wavelength range of $430-600 \mathrm{~nm}$ (Figure 3a). Dopamine oxidation produces oligomers, which self-assemble and form PDA through $\pi-\pi^{*}$ stacking interactions. ${ }^{19,20}$ Increased $\pi-\pi^{*}$ interactions result in aggregation-induced quenching in the course of time (Figure S3). ${ }^{21}$ When treated with $\mathrm{H}_{2} \mathrm{O}_{2}$, PDA fluorescence is found to turn on due to decomposition of PDA polymer to nanoaggregates by $\mathrm{H}_{2} \mathrm{O}_{2} \cdot{ }^{22-24}$ Accordingly, PDA fluorescence was increased with increasing glucose concentration due to $\mathrm{H}_{2} \mathrm{O}_{2}$ generated through glucose oxidation (Figure 3a,b). Furthermore, enhancement of PDA fluorescence was also observed by the naked eye under UV light (Figure 3c). Interestingly, fluorescence maximum was shifted to blue $(470 \mathrm{~nm})$ in the presence of glucose with concentration above $5 \mathrm{mM}$, revealing reduction of nanoaggregate size with gradual increase of $\mathrm{H}_{2} \mathrm{O}_{2} .{ }^{25}$ We investigated the effect of $\mathrm{H}_{2} \mathrm{O}_{2}$ on PDA fluorescence. Besides increasing fluorescence intensity, fluorescence maximum was observed to shift from 500 to $470 \mathrm{~nm}$ with increasing $\mathrm{H}_{2} \mathrm{O}_{2}$ (Figure S4). The limit of detection of fluorescence-based glucose assay was calculated to be $130 \mu \mathrm{M}$ by plotting the linear calibration curve (inset of Figure $3 \mathrm{~b}$ ).

Although fluorescence enhancement was also observed in bulk liquid, where insignificant evaporation occurs, the reaction was much slower. To investigate the same reaction in bulk, fluorescence intensities were recorded after incubating PDA with glucose/GOx in $4 \mathrm{~mL}$ of buffer solutions at $37^{\circ} \mathrm{C}$. Figure 4 shows time dependence of fluorescence intensity at $500 \mathrm{~nm}$. During the initial stages of PDA growth, dopamine emission centered at $460 \mathrm{~nm}$ was observed besides PDA emission centered around $500 \mathrm{~nm}$ and then gradually disappeared (Figure S5). After the addition of dopamine to oxidized 


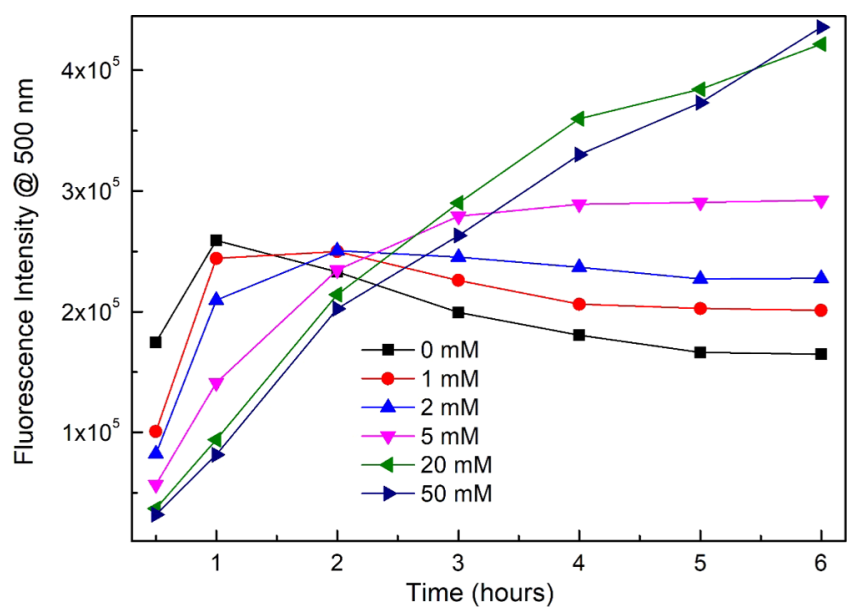

Figure 4. Time dependence of fluorescence intensities of PDA at 500 $\mathrm{nm}$ after incubation with glucose with concentration ranging from 1 to $50 \mathrm{mM}$ in bulk solutions. The complete spectrum for all incubation times is given in the Supporting Information.

glucose, different fluorescence intensities were recorded for different glucose concentrations. However, enhancement response was observed with increasing glucose concentration only after $4 \mathrm{~h}$ incubation (Figure 4 ). Until $4 \mathrm{~h}$ incubation, there is no correlation between the glucose concentration and fluorescence intensity at $500 \mathrm{~nm}$. This result can be explained by the suppression of PDA formation soon after dopamine addition to oxidized glucose when $\mathrm{H}_{2} \mathrm{O}_{2}$ amount is high. Reorganization of PDA nanoclusters through $\pi-\pi^{*}$ stacking interactions in the course of time resulted in fluorescence enhancement. Droplet-based assay accelerated reaction between PDA and $\mathrm{H}_{2} \mathrm{O}_{2}$, and reduced the required time to $50 \mathrm{~min}$ from $4 \mathrm{~h}$ to generate fluorescent enhancement for a wide range of glucose concentration from 1 to $50 \mathrm{mM}$. Dopamine emission was not observed in evaporating droplets due to high PDA growth rate. It is important to note that shift of fluorescence maximum was not observed in the bulk liquid, revealing the inefficient interaction with $\mathrm{H}_{2} \mathrm{O}_{2}$.

Besides glucose detection, patterned surfaces were also used for droplet-based DNA detection. First, probe DNA was covalently attached to wetted patterns using silane chemistry (see Materials and Methods section for details). Briefly, wetted patterns were first epoxylated with (3-glycidoxypropyl)trimethoxysilane (GOPTS) and then amine-ended DNA molecules were anchored to epoxy groups in sodium carbonate buffer with $\mathrm{pH} 9$ (Figure 5a). Surfaces were washed with bovine serum albumin (BSA) solution to remove the unbound DNA molecules. Binding of GOPTS and DNA to UV/ozonetreated patterns was analyzed by X-ray photoelectron spec-
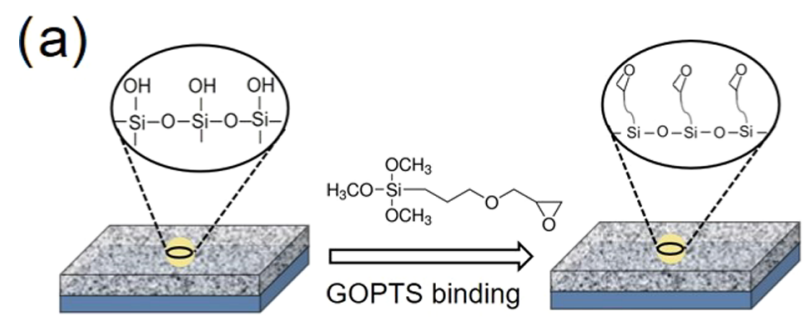

。
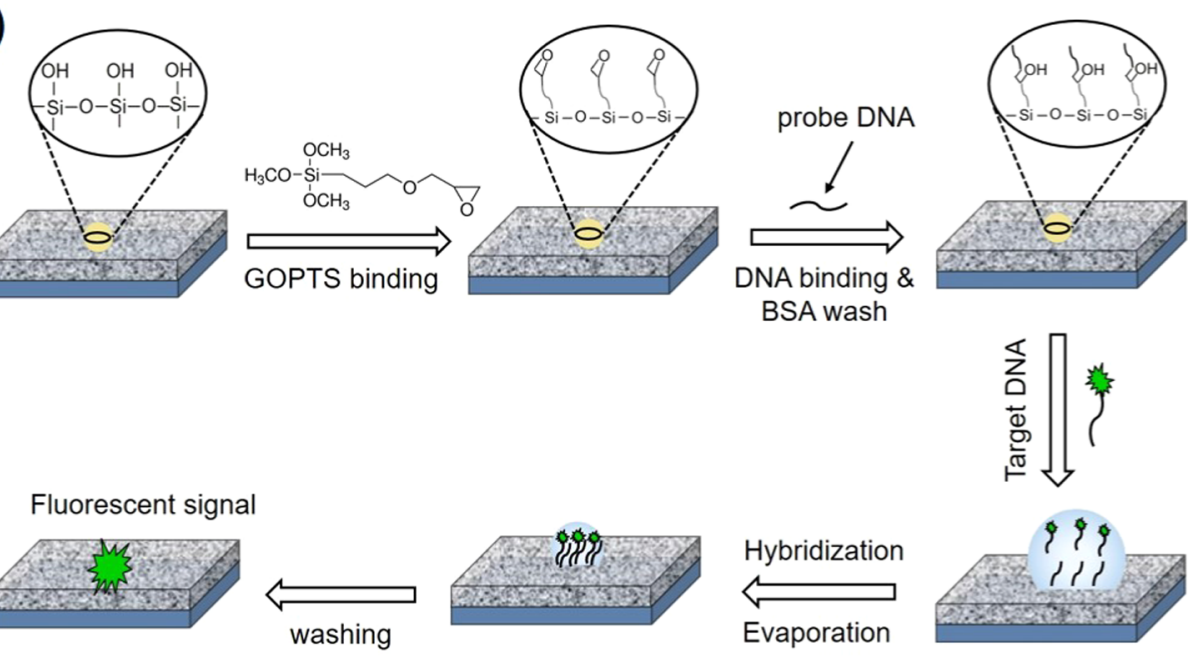

Hybridization

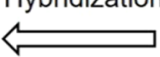

Evaporation

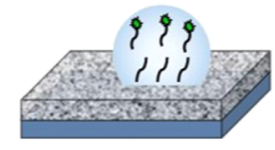



Figure 5. (a) Schematic representation of epoxysilane binding onto UV/ozone-treated regions, followed by attachment of probe DNA, hybridization of fluorescence-tagged target DNA, evaporation-induced analyte enrichment, and fluorescence enhancement. (b) Confocal image of wetted pattern after the attachment of Cy5-tagged probe DNA. (c) Confocal image of wetted pattern after hybridization of probe DNA with 20 pM Cy3-tagged target DNA in $4 \mu \mathrm{L}$ of evaporating droplet. 
troscopy. C 1s spectrum of UV/ozone-treated areas showed the decomposition of $\mathrm{CH}_{3}$ groups (Figure S6). Peaks at 286.7 and $287.7 \mathrm{eV}$ confirmed epoxysilane binding, whereas peaks at 285.4 and $286.6 \mathrm{eV}$ and increase of carbon intensities revealed DNA binding (Figure S7). ${ }^{26}$ Binding of probe DNA was also monitored with confocal microscopy using Cy5 tagging. Red emission of $\mathrm{Cy} 5$ collected from porous ormosil network shows that probe DNA uniformly bonded all over the epoxyfunctionalized silica nanoparticles on wetted pattern (Figure $5 b)$.

Droplets $(4 \mu \mathrm{L})$ including Cy3-tagged $200 \mathrm{fM}, 20 \mathrm{pM}$, and $200 \mathrm{pM}$ target DNA were added to probe-bound patterns and left to incubate while evaporating in the meantime. After washing with BSA and phosphate-buffered saline (PBS) solutions, emission intensities were analyzed with confocal microscopy. Figure 5c shows fluorescence signal collected from a spot hybridized with $20 \mathrm{pM}$ target DNA. In addition, hybridization was performed in bulk solution. A patterned surface with probe DNA anchored on $0.9 \mathrm{~mm}$ pattern was immersed in $2 \mathrm{~mL}$ of $20 \mathrm{pM}$ target DNA solution and left to incubate. Fluorescence signal obtained in droplet-based assay was 2 orders of magnitude higher than that in solution-based assay (Figure 6). Hybridization signal for noncomplementary

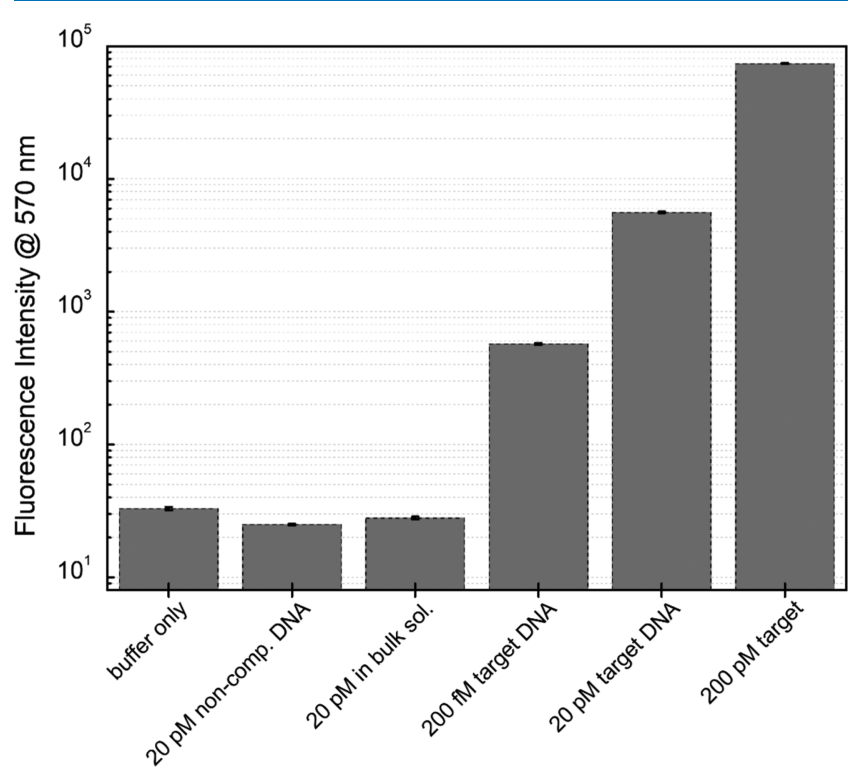

Figure 6. Fluorescence intensities measured from confocal images of patterns incubated with droplets containing buffer solution without DNA; 20 pM noncomplementary DNA; and 20 pM, $200 \mathrm{fM}$, and 200 pM complementary DNA. Additionally, fluorescence of wetted pattern incubated in $4 \mathrm{~mL}$ bulk solution of $20 \mathrm{pM}$ complementary DNA.

DNA was at the same level with buffer solution revealing the specificity of the biosensor for target DNA (Figure 6). Detection of DNA as low as $200 \mathrm{fM}$ was achieved in the assay.

\section{CONCLUSIONS}

In conclusion, we fabricated a simple detection platform for glucose and DNA assays by using superhydrophilic-superhydrophobic patterned surfaces. A simple UV/ozone-based chemical treatment was used to generate wetted patterns on superhydrophobic coatings produced via sol-gel method. Aqueous droplets with very small volume can be confined on wetted patterns. Analytes dissolved in droplets can be enriched upon evaporation of microdroplets, which are confined on wetted patterns. Polydopamine (PDA) was used as a single fluorescent probe for glucose, for the first time in this study, by benefitting from $\mathrm{H}_{2} \mathrm{O}_{2}$-dependent fluorescent enhancement of PDA. A glucose assay with reduced response time and increased sensitivity was obtained due to enrichment-induced increase of reaction rates. Contrary to droplet-based assay, fluorescence enhancement was very slow in bulk solutions. Glucose detection with concentration ranging from 1 to 50 $\mathrm{mM}$ was shown using bright PDA fluorescence, which can also be observed by the naked eye. PDA with its biocompatibility and promising response curve shows potential for replacing the $\mathrm{HRP} /$ chromogen indicators in glucose sensors, especially for wearable and implantable sensors. Furthermore, wetted patterns were functionalized with DNA strands and were used for droplet-based DNA analysis using very low sample volumes. Target DNA with concentration as low as $200 \mathrm{fM}$ can be detected by means of enrichment effect. The presented versatile patterned surfaces with their simple production scheme have potential for a wide range of biochemical analysis.

\section{MATERIALS AND METHODS}

Materials. Methyltrimethoxysilane (MTMS), oxalic acid, ammonium hydroxide (25\%), sodium hydroxide, ethanol, glucose, and hydrochloric acid were purchased from Merck. Methanol was purchased from Carlo-Erba. Glucose oxidase $(\mathrm{GOx})$, dopamine hydrochloride, tris(hydroxymethyl)aminomethane, phosphate-buffered saline (PBS) tablets, (3glycidoxypropyl)trimethoxysilane (GOPTS), sodium carbonate, and bovine serum albumin (BSA) were purchased from Sigma-Aldrich. Molecular biology (MB) grade water was purchased from Fisher Scientific. All chemicals were used as received. Amino-modified, $\mathrm{Cy} 5 / \mathrm{Cy} 3$-tagged, and tag-free single-stranded DNA (ss-DNA) sequences were purchased from Sentegen Biotech. Sequences were as follows:

- Probe DNA: 5'-C6-Amino-TTGGAACATTC-Cy5-3' (fluorescence-tagged) 5'-C6-Amino-TTGGAACATTC-3' (tag-free)

- Target DNA: 5'-Cy3-GAATGTTCCAA-3'

- Noncomplementary DNA: 5'-Cy3-TGCCGCTGCAC$3^{\prime}$

Preparation of Superhydrophilic-Superhydrophobic Patterned Surfaces. Superhydrophobic coatings were prepared using previously reported sol-gel method. . $^{9}$ MTMS $(1 \mathrm{~mL})$ was dissolved in $9.74 \mathrm{~mL}$ of methanol. Following 15 min stirring, $0.5 \mathrm{~mL}$ of $1 \mathrm{mM}$ oxalic acid solution was added dropwise and the solution was gently stirred for $30 \mathrm{~min}$. Then, the mixture was left to hydrolyze organosilane precursor completely for $24 \mathrm{~h}$ at room temperature. After the hydrolysis step, $0.19 \mathrm{~mL}$ of water and $0.42 \mathrm{~mL}$ of ammonia solution $(25 \%)$ were added and gently stirred for $15 \mathrm{~min}$. The resultant mixture was left for 2 days at room temperature to obtain a complete gel. After the aging step, $9 \mathrm{~mL}$ of methanol was added, followed by homogenization using an ultrasonic liquid processor for $45 \mathrm{~s}$ at $20 \mathrm{~W}$. Portions of resultant colloidal silica solution $(250 \mathrm{~mL})$ were spin-coated onto clean quartz or glass substrates $\left(1 \times 2 \mathrm{~cm}^{2}\right)$ at $3000 \mathrm{rpm}$ for $45 \mathrm{~s}$. The as-prepared surfaces were covered with cellulose acetate sheets, on which circular holes with 0.9 or $1.4 \mathrm{~mm}$ diameter were formed using a $30 \mathrm{~W} \mathrm{CO}_{2}$ laser cutter system (Epilog Zing). Covered surfaces were exposed to UV/ozone for $60 \mathrm{~min}$ using a surface cleaning system (PSD-UV, Novascan Technologies) to convert exposed 
superhydrophobic regions to superhydrophilic. The power intensities of the UV/ozone cleaner were 8 and $30 \mathrm{~mW} / \mathrm{cm}^{2}$ for 185 and $253.7 \mathrm{~nm}$ wavelengths, respectively.

Characterizations. Static water contact angles of surfaces were measured with a contact angle meter (OCA 30, DataPhysics). Water droplets $(20 \mu \mathrm{L})$ were used for the measurements. The Laplace-Young equation was used for contact angle calculations. Surface morphology was examined using a scanning electron microscope (Quanta 200F, FEI). Surface chemistry was characterized by X-ray photoelectron spectroscopy (K-Alpha, Thermo Scientific).

Preparation of Buffer Solutions. PBS (100 mM) was prepared by dissolving 10 PBS tablets in $200 \mathrm{~mL}$ of deionized water, and the solution $\mathrm{pH}$ was adjusted to 7.4 using $1 \mathrm{M}$ sodium hydroxide solution. Tris solution $(50 \mathrm{mM})$ was prepared and its $\mathrm{pH}$ was adjusted to 8.6 using $2 \mathrm{M}$ hydrochloric acid. Sodium carbonate buffer $(100 \mathrm{mM})$ was prepared and its $\mathrm{pH}$ was adjusted to 9.0 using $2 \mathrm{M}$ hydrochloric acid.

Glucose Assay. PBS solutions (2 mL, pH 7.4), including glucose with concentrations varying from 1 to $50 \mathrm{mM}$, were mixed with $50 \mu \mathrm{L}$ of $1 \mathrm{mg} / \mathrm{mL}$ GOx solution. Portions of the mixtures $(20 \mu \mathrm{L})$ were drop-cast onto separate superwetted spots of patterned surfaces and incubated at $37{ }^{\circ} \mathrm{C}$ for $30 \mathrm{~min}$. For bulk assay, the solutions were directly incubated at $37{ }^{\circ} \mathrm{C}$ for $30 \mathrm{~min}$. Dopamine in Tris- $\mathrm{HCl}$ buffer $(\mathrm{pH} 8.6)$ with a final dopamine concentration of $0.5 \mathrm{mM}$ was added to the glucose-GOx mixtures. For droplet assay, $20 \mu \mathrm{L}$ portions of dopamine were added and incubated until droplets were completely evaporated, which took approximately $50 \mathrm{~min}$. The assay time is a function of the hydrophilic spot size, droplet size, and evaporation rate, which should be optimized for any given assay. For bulk assay, dopamine and glucose-GOx mixtures were incubated at $37^{\circ} \mathrm{C}$ up to $5 \mathrm{~h}$. A control sample containing GOx enzyme without glucose was also prepared and incubated as all other samples.

DNA Assay. Patterned surfaces were immersed in ethanol solution containing $2 \%(\mathrm{v} / \mathrm{v})$ GOPTS and incubated at $37{ }^{\circ} \mathrm{C}$ overnight. Then, the surfaces were washed with ethanol several times, followed by drying at $120^{\circ} \mathrm{C}$ for $1 \mathrm{~h}$. Portions $(4 \mu \mathrm{L})$ of $1 \mu \mathrm{M}$ probe DNA (Cy5-tagged or tag-free) in sodium carbonate buffer solutions ( $\mathrm{pH} 9.0$ ) were drop-cast onto superwetted spots. After incubation at $37^{\circ} \mathrm{C}$ for $4 \mathrm{~h}$ in a closed chamber humidified around $80 \%$, the surfaces were washed with PBS solutions ( $\mathrm{pH} 7.4$ ) to remove the unbound probe DNA strands. The surfaces were then washed with PBS, followed by washing with $1 \%$ BSA-PBS solution and MB grade water. Target DNA ( $4 \mu \mathrm{L} ; 200 \mathrm{fM}, 20 \mathrm{pM}$, and $200 \mathrm{pM}$ ) in PBS solutions were drop-cast onto probe-bound spots. Microdroplets were left to incubate in the dark at $37^{\circ} \mathrm{C}$ for $2 \mathrm{~h}$ in a closed chamber with $40 \%$ humidity. The surfaces were then washed with PBS ( $\mathrm{pH} 7.4$ ) and MB grade water and dried with nitrogen blow.

FluorescenceMeasurements. Fluorescence intensities of droplets and bulk glucose assays were analyzed using a UV lamp (366 nm, 8W, CAMAG) and a fluorescence spectrophotometer (HORIBA, Fluorolog-3, $450 \mathrm{~W}$ ) by exciting samples at $400 \mathrm{~nm}$ and collecting intensities within the $420-600 \mathrm{~nm}$ range. Fluorescent images of droplet-based DNA assay were taken using a confocal microscope (LSM 510, Zeiss using 10X objective. Helium lasers at 543 and $633 \mathrm{~nm}$ were used for excitation. Emissions at around 570 and $650 \mathrm{~nm}$ were collected for $\mathrm{Cy} 3$ and $\mathrm{Cy} 5$, respectively.

\section{ASSOCIATED CONTENT}

\section{Supporting Information}

The Supporting Information is available free of charge on the ACS Publications website at DOI: 10.1021/acsomega.8b00389.

Water droplet profile on superhydrophobic surface; change of water contact angle of ormosil surfaces depending on UV/ozone treatment time; time-dependent fluorescence spectra of polydopamine; fluorescence spectra of PDA incubated with $\mathrm{H}_{2} \mathrm{O}_{2}$; fluorescence spectra of PDA after incubation with glucose/GOx in bulk solutions; $\mathrm{C} 1 \mathrm{~s}$ spectra of untreated superhydrophobic surface; C 1s spectra of UV/ozone-treated surface; C 1s spectra of epoxylated patterns; and C 1s spectra of DNA-functionalized patterns (PDF)

\section{AUTHOR INFORMATION}

\section{Corresponding Author}

*E-mail: elbuken@unam.bilkent.edu.tr. Phone: +90 312290 3550.

ORCID $\odot$

Mehmet Bayindir: 0000-0003-0233-6870

Caglar Elbuken: 0000-0001-8359-6871

Notes

The authors declare no competing financial interest.

\section{ACKNOWLEDGMENTS}

P.B. was supported by TÜBİTAK-BİDEB graduate fellowship. This study is partly supported by TÜBITAK under the Project no. 111 T696.

\section{REFERENCES}

(1) Liu, W.-D.; Yang, B. Patterned Surfaces for Biological Applications: A New Platform Using Two Dimensional Structures as Biomaterials. Chin. Chem. Lett. 2017, 28, 675-690.

(2) Zhang, Y.; Ren, T.; Li, T.; He, J.; Fang, D. Paper-Based Hydrophobic/Lipophobic Surface for Sensing Applications Involving Aggressive Liquids. Adv. Mater. Interfaces 2016, 3, No. 1600672.

(3) Yang, S.; Dai, X.; Stogin, B. B.; Wong, T.-S. Ultrasensitive Surface-Enhanced Raman Scattering Detection in Common Fluids. Proc Natl Acad Sci U.S.A. 2016, 113, 268-273.

(4) Xu, L. P.; Chen, Y.; Yang, G.; Shi, W.; Dai, B.; Li, G.; Cao, Y.; Wen, Y.; Zhang, X.; Wang, S. Ultratrace DNA Detection Based on the Condensing-Enrichment Effect of Superwettable Microchips. Adv. Mater. 2015, 27, 6878-6884.

(5) Fukada, K.; Kawamura, N.; Shiratori, S. Trace Material Capture by Controlled Liquid Droplets on a Superhydrophobic/Hydrophilic Surface. Anal. Chem. 2017, 89, 10391-10396.

(6) Wu, T.; Xu, T.; Chen, Y.; Yang, Y.; Xu, L. P.; Zhang, X.; Wang, S. Renewable Superwettable Biochip for miRNA Detection. Sens. Actuators, B 2018, 258, 715-721.

(7) Chen, Y.; Xu, L. P.; Meng, J.; Deng, S.; Ma, L.; Zhang, S.; Zhang, X.; Wang, S. Superwettable Microchips with Improved Spot Homogeneity Toward Sensitive Biosensing. Biosens. Bioelectron. 2018, 102, 418-424.

(8) Chen, Y.; Min, X.; Zhang, X.; Zhang, F.; Lu, S.; Xu, L.-P.; Lou, X.; Xia, F.; Zhang, X.; Wang, S. AIE-based Superwettable Microchips for Evaporation and Aggregation Induced Fluorescence Enhancement Biosensing. Biosens. Bioelectron. 2018, 111, 124-130.

(9) Beyazkilic, P.; Tuvshindorj, U.; Yildirim, A.; Elbuken, C.; Bayindir, M. Robust Superhydrophilic Patterning of Superhydrophobic Ormosil Surfaces for High-throughput On-Chip Screening Applications. RSC Adv. 2016, 6, 80049-80054. 
(10) Budunoglu, H.; Yildirim, A.; Guler, M.; Bayindir, M. Highly Transparent, Flexible, and Thermally Stable Superhydrophobic ORMOSIL Aerogel Thin Films. ACS Appl. Mater. Interfaces 2011, 3, 539-545.

(11) Hernandez-Perez, R.; Fan, Z. H.; Garcia-Cordero, J. L. Evaporation-Driven Bioassays in Suspended Droplets. Anal. Chem. 2016, 88, 7312-7317.

(12) He, F.; Tang, Y.; Yu, M.; Wang, S.; Li, Y.; Zhu, D. Fluorescence-Amplifying Detection of Hydrogen Peroxide with Cationic Conjugated Polymers, and Its Application to Glucose Sensing. Adv. Funct. Mater. 2006, 16, 91-94.

(13) He, F.; Feng, F.; Wang, S.; Li, Y.; Zhu, D. Fluorescence ratiometric assays of hydrogen peroxide and glucose in serum using conjugated polyelectrolytes. J. Mater. Chem. 2007, 17, 3702-3707.

(14) Zhang, T.; Fan, H.; Liu, G.; Jiang, J.; Zhou, J.; Jin, Q. Different effects of $\mathrm{Fe} 2+$ and $\mathrm{Fe} 3+$ on conjugated polymer $\mathrm{PPESO}_{3}$ : a novel platform for sensitive assays of hydrogen peroxide and glucose. Chem. Commun. 2008, 5414-5416.

(15) Shen, X.; Shi, Y.; Peng, B.; Li, K.; Xiang, J.; Zhang, G.; Liu, Z.; Chen, Y.; Zhang, D. Fluorescent Polymeric Micelles with Tetraphenylethylene Moieties and Their Application for the Selective Detection of Glucose. Macromol. Biosci. 2012, 12, 1583-1590.

(16) Yildirim, A.; Bayindir, M. Turn-on Fluorescent Dopamine Sensing Based on in Situ Formation of Visible Light Emitting Polydopamine Nanoparticles. Anal. Chem. 2014, 86, 5508-5512.

(17) Xiong, B.; Chen, Y.; Shu, Y.; Shen, B.; Chan, H. N.; Chen, Y.; Zhou, J.; Wu, H. Highly emissive and biocompatible dopaminederived oligomers as fluorescent probes for chemical detection and targeted bioimaging. Chem. Commun. 2014, 50, 13578-13580.

(18) Quignard, S.; d'Ischia, M.; Chen, Y.; Fattaccioli, J. UltravioletInduced Fluorescence of Polydopamine-Coated Emulsion Droplets. ChemPlusChem 2014, 79, 1254-1257.

(19) Liu, Y.; Ai, K.; Lu, L. Polydopamine and Its Derivative Materials: Synthesis and Promising Applications in Energy, Environmental, and Biomedical Fields. Chem. Rev. 2014, 114, 5057-5115.

(20) Hong, S.; Na, Y. S.; Choi, S.; Song, I. T.; Kim, W. Y.; Lee, H. Non-Covalent Self-Assembly and Covalent Polymerization CoContribute to Polydopamine Formation. Adv. Funct. Mater. 2012, 22, 4711-4717.

(21) Yu, X.; Fan, H.; Liu, Y.; Shi, Z.; Jin, Z. Characterization of Carbonized Polydopamine Nanoparticles Suggests Ordered Supramolecular Structure of Polydopamine. Langmuir 2014, 30, 54975505.

(22) Zhang, X.; Wang, S.; Xu, L.; Feng, L.; Ji, Y.; Tao, L.; Li, S.; Wei, Y. Biocompatible Polydopamine Fluorescent Organic Nanoparticles: Facile Preparation and Cell Imaging. Nanoscale 2012, 4, 5581-5584.

(23) Chen, X.; Yan, Y.; Müllner, M.; Van Koeverden, M. P.; Noi, K. F.; Zhu, W.; Caruso, F. Engineering Fluorescent Poly(dopamine) Capsules. Langmuir 2014, 30, 2921-2925.

(24) Lin, J.-H.; Yu, C.-J.; Yanga, Y.-C.; Tseng, W.-L. Formation of Fluorescent Polydopamine Dots from Hydroxyl Radical-Induced Degradation of Polydopamine Nanoparticles. Phys. Chem. Chem. Phys. 2015, 17, 15124-15130.

(25) Bruchez, M., Jr.; Moronne, M.; Gin, P.; Weiss, S.; Alivisatos, A. P. Semiconductor Nanocrystals as Fluorescent Biological Labels. Science 1998, 281, 2013-2016.

(26) Escorihuela, J.; Bañuls, M. J.; Puchades, R.; Maquieira, A. DNA Microarrays on Silicon Surfaces through Thiol-Ene Chemistry. Chem. Commun. 2012, 48, 2116-2118. 